\title{
GRAPPA Trainees Symposium 2018: A Report from the GRAPPA 2018 Annual Meeting
}

\author{
Adam R. Ford, Enrico Mascia, Wolf-Henning Boehncke, and Christopher T. Ritchlin
}

\begin{abstract}
The Group for Research and Assessment of Psoriasis and Psoriatic Arthritis (GRAPPA) held a trainee symposium at its 2018 annual meeting in Toronto, Ontario, Canada. Rheumatology and dermatology trainees engaged in psoriasis or psoriatic arthritis research presented their work. This report briefly reviews 5 oral presentations and 21 posters presented at the meeting. (J Rheumatol Suppl. 2019 June;95:4-10; doi:10.3899/jrheum.190123)
\end{abstract}

Key Indexing Terms: GRAPPA TRAINEE
PSORIASIS

RHEUMATOLOGIST

\section{PSORIATIC ARTHRITIS DERMATOLOGIST}

The Group for Research and Assessment of Psoriasis and Psoriatic Arthritis (GRAPPA) held this year's trainee symposium at its 2018 annual meeting in Toronto, Ontario, Canada. As in past years, rheumatology or dermatology trainees from North America, South America, and Europe, who are current GRAPPA members or who were nominated by GRAPPA members, were invited to submit abstracts based on recent research in psoriatic arthritis (PsA) or psoriasis ${ }^{1-9}$. A total of 54 abstracts were submitted and ranked by a committee of reviewers. Five trainees with the highest-scored abstracts were invited to deliver oral presentations, and 21 trainees presented posters that outlined key aspects of their research. Dr. Christopher T. Ritchlin (Rochester, New York, USA) and Dr. Wolf-Henning Boehncke (Geneva, Switzerland) co-chaired the symposium in which GRAPPA members discussed the findings that were presented by trainees and suggested how trainees might further develop their current research projects.

From the Keck School of Medicine of the University of Southern California, Los Angeles, California; Division of Allergy, Immunology, and Rheumatology, University of Rochester Medical Center, Rochester, New York, USA; University of Cagliari, Rheumatology Department at Policlinico Universitario, Monserrato (Cagliari), Italy; Department of Dermatology, Division of Dermatology and Venereology, Geneva University Hospitals; Department of Pathology and Immunology, University of Geneva, Geneva, Switzerland.

As part of the supplement series GRAPPA 2018, this report was reviewed internally and approved by the Guest Editors for integrity, accuracy, and consistency with scientific and ethical standards.

A.R. Ford, BS, Medical Student, Keck School of Medicine of the University of Southern California; E. Mascia, Resident of Rheumatology, University of Cagliari, Rheumatology Department at Policlinico Universitario; W.H. Boehncke, MD, Professor and Chair, Department of Dermatology, Division of Dermatology and Venereology, Geneva University Hospitals, and Department of Pathology and Immunology, University of Geneva; C.T. Ritchlin, MD, MPH, Professor of Medicine, Division of Allergy, Immunology, and Rheumatology, University of Rochester Medical Center. Address correspondence to Dr. C.T. Ritchlin, Division of Allergy, Immunology, and Rheumatology, University of Rochester Medical Center, 601 Elmwood Ave., Box 695, Rochester, New York 14642, USA.

E-mail: Christopher_Ritchlin@URMC.Rochester.edu

\section{Oral Presentations}

Online care is as effective as in-person care for improving quality of life and mental health in psoriasis: results from a randomized controlled equivalency trial (Adam R. Ford, Los Angeles, California, USA).

There is a lack of access to dermatologists in the United States. Connected health is a model of healthcare delivery that uses technology to provide healthcare remotely and has the potential to increase access to specialty care. This 12-month randomized controlled equivalency trial evaluated whether an online model for psoriasis results in equivalent improvements in quality of life (QOL) and mental health compared to in-person care.

Adult patients with psoriasis $(n=300)$ were randomized to an online intervention or in-person care. The online model enabled patients and primary care providers to access dermatologists online. The in-person group sought care in person. Data were collected at baseline and at 3-month intervals. QOL was measured using Skindex-16 and Dermatology Life Quality Index (DLQI), and depression severity was measured using Patient Health Questionnaire-9 (PHQ-9). Both groups demonstrated improvement in all outcome measures from baseline across followup visits. In the adjusted model using repeated measures analysis, the average difference in Skindex-16 between the 2 groups was -0.83 (95\% CI -5.18 to 3.51$)$, which was within the prespecified equivalence margin $( \pm 7.0)$. The average difference in DLQI between the 2 groups was -0.45 (95\% CI -1.29 to 0.38$)$, which was within the prespecified equivalence margin $( \pm 2.5)$. The average difference in PHQ-9 between the 2 groups was -0.33 (95\% CI -1.20 to 0.55$)$, which was within the prespecified equivalence margin $( \pm 3.0)$. The author concluded that the online model for psoriasis was as effective as in-person care at improving QOL and mental health outcomes.

Genetic variants in the TNF- $\alpha$ region: a novel biomarker of clinical response to anti-TNF- $\alpha$ drugs in PsA patients (Enrico Mascia, Cagliari, Italy). 
This study searched for possible genetic variants in the entire tumor necrosis factor- $\alpha$ (TNF- $\alpha$ ) genomic region that predict therapeutic response to anti-TNF- $\alpha$ drugs in a group of patients from Sardinia. Of 130 identified patients with PsA, 70 were treated with anti-TNF- $\alpha$ drugs and were scanned using the next-generation sequencing technique, which focuses on the TNF region. Response to anti-TNF- $\alpha$ drugs was evaluated according to PsA Response Criteria and American College of Rheumatology 20\% improvement criteria (ACR20). For the 70 patients, preliminary data showed $53(76 \%)$ responding and $17(24 \%)$ not responding to treatment. There were 65 single-nucleotide polymorphisms (SNP) without a direct link with allele distribution and susceptibility for PsA. The study compared allele and genotype frequencies in the 2 groups and showed a significant association of SNP $-376 \mathrm{G}>\mathrm{A}$ with the response to anti-TNF- $\alpha$ treatment $(\mathrm{p}=0.005)$. This SNP is located between the lymphotoxin- $\alpha$ precursor and TNF genes, and may have regulatory functions in the expression of the 2 genes. Analysis of the region showed that the allele of SNP $-376 \mathrm{G}>\mathrm{A}$ is in linkage disequilibrium with TNF promoter SNP alleles that seem to influence baseline TNF expression levels in the carrier individuals. The author concluded that SNP -376G $>$ A in PsA has potential to predict response to treatment with anti-TNF- $\alpha$ drugs. This SNP has not been studied in patients with PsA from other geographical areas. It could therefore represent a novel biomarker of response to therapy and could prove useful from a precision medicine approach, and it could also lead to cost savings.

How to define remission and low disease activity in PsA? An analysis of 439 patients with a double perspective (Déborah Puyraimond-Zemmour, Paris, France).

Although remission or low disease activity (LDA) is the treatment goal in PsA, there is no consensus on what defines remission/LDA. This cross-sectional study was performed in 14 countries on 445 consecutive adults with definite PsA. It assessed the frequency of remission/LDA using different definitions, evaluated agreement between these definitions, and described the characteristics of patients in remission from patients' and physicians' perspectives.

Remission was defined from the physicians' perspective as very low disease activity (VLDA), Disease Activity Index for PsA (DAPSA) < 4, clinical DAPSA $<4$, and physician-perceived remission (yes/no), and from the patients' perspective as patient's global assessment $<1$ and patient-perceived remission. LDA was defined from the physicians' perspective as minimal disease activity (MDA), DAPSA $<14$, clinical DAPSA $<1$, modified GRAPPA Composite Exercise index $<2.3$, and physician-perceived LDA, and from the patients' perspective as Patient-Acceptable Symptom State (PASS) and patient-perceived LDA. Proportions achieving each remission/LDA criterion were calculated, and agreement between definitions was assessed using $\kappa$.
The frequency of remission varied from $12.1 \%$ (VLDA) to $39.2 \%$ (physician-perceived remission); the frequency of LDA varied from $26.0 \%$ (MDA) to $67.0 \%$ (PASS). There was good agreement between physicians' composite scores for remission ( $\kappa$ DAPSA/VLDA 0.56, clinical DAPSA/VLDA 0.57). Agreement was moderate to good between physicians' and patients' scores for remission. Agreement was lower among all definitions for LDA.

The characteristics of patients in remission differed depending on the patients' and physicians' perspectives. Physician-perceived remission/LDA does not agree well with composite scores or patient perspectives in PsA.

Tc17 CD8+ MAIT cells: a new dimension for the IL-23/IL-17 cytokine network in PSA (Kanika Sood, Sacramento, California, USA).

Mucosal-associated invariant T (MAIT) cells are relevant for autoimmune diseases because of their production of inflammatory cytokines. Their gut origin, lineage toward interleukin (IL)-23/IL-17 signature cytokines, and cytotoxic $\mathrm{T}$ cell effector phenotype suggest that this population may be more relevant for PsA compared to rheumatoid arthritis (RA). The authors investigated the pathological significance of MAIT cells in PsA regarding their function and regulatory role of IL-23/IL-23 receptor (IL-23R). Peripheral blood mononuclear cells and synovial fluid mononuclear cells (SFMC) were collected from 10 patients, each with active untreated PsA, RA, or osteoarthritis (OA), and age- and sex-matched controls. The study used the phorbol myristate acetate/inomycin activation method and a standardized method of Th17 cell enrichment with recombinant IL-23 to culture and enrich the MAIT cells. PsA SFMC MAIT cells produced significantly more IL-17 (32.66 $\pm 4.01 \%)$ compared to RA SFMC $(23.93 \pm 2.81 \%)$ and OA SFMC $(5.02 \pm 0.16 \% ; \mathrm{p}<0.05)$. IL-17+ MAIT cells were predominantly CD8+ (about 90\%). Compared to conventional $\alpha \beta \mathrm{T}$ cells, in which CD4+ T cells are the major source of IL-17, it was observed that MAIT cells in SFMC of PsA were predominantly CD8+. Tc17 CD8+/MAIT cells are more relevant for PsA because of their MHC class 1 association. MAIT cells express functionally active IL-23R, which provides a new mechanism by which IL-23 can regulate these cells once they migrate to joint tissue.

A Western (high-fat, high-glucose) diet predisposes mice to enhanced susceptibility to imiquimod-induced psoriatic skin changes compared to a high-fat alone diet and control diets (Sebastian Yu, Sacramento, California, USA).

A high-fat and high-sugar diet [i.e., a Western diet (WD)] is involved in the development of obesity, a psoriatic comorbidity. Authors compared WD-fed and high-fat diet (HFD)-fed mice and examined whether these diets have distinct effects on the susceptibility to imiquimod (IMQ)-induced psoriasiform dermatitis (PsD). C57BL/6 mice

Personal non-commercial use only. The Journal of Rheumatology Copyright (C) 2019. All rights reserved. 
were fed with a WD, a control diet (CD), an HFD, or a low-fat diet for up to 16 weeks. IMQ cream $(5 \%)$ or vehicle cream was then applied to both ears of each mouse for 5 consecutive days to induce PsD. Ear thickness was measured daily. The results showed differences in ear thickness change at Day 5 between WD and CD groups (Week 12: $0.211 \mathrm{~mm}$ vs $0.156 \mathrm{~mm}, \mathrm{p}=0.014$; Week $16: 0.211 \mathrm{~mm}$ vs $0.138 \mathrm{~mm}$, $\mathrm{p}=0.005)$. The ear thickness change of IMQ-treated WD mice was also significantly higher than that of IMQ-treated HFD mice at Day 5 (Week 12: $\mathrm{p}=0.009$; Week 16: $\mathrm{p}=0.004)$. Quantitative real-time PCR (qRT-PCR) revealed that after feeding for 12 weeks, the baseline expression levels of IL-17A and IL-17F were 43.9-fold ( $\mathrm{p}=0.007)$ and 14.2-fold $(\mathrm{p}=0.001)$ higher, respectively, in the WD group compared with the CD group. The authors concluded that both the WD and the HFD contributed to weight gain, with HFD-treated mice gaining more weight, but only WD-fed mice showed enhanced susceptibility to PsD. This suggests that obesity alone is not sufficient to promote inflammatory changes in the skin.

\section{Poster Presentations}

Hussein Al-Mossawi (Oxford, UK) performed a prospective study aimed at interrogating ex vivo synovial fluid (SF) and matched blood in the large joint oligo variant of PsA using time-of-flight mass cytometry (CyTOF) followed by an RNA transcriptomic analysis of key sorted immune populations. Paired blood and SF were freshly isolated and stained for CyTOF. Key expanded populations were freshly sorted from the blood and SF using flow sorting and were immediately preserved in lysis buffer. The extracted RNA was studied using a 384-gene array and a subsequent pathway analysis was performed. The SF in PsA is fundamentally different compared to the matched peripheral blood with a significant expansion of several populations. In particular, the authors observed a significant expansion of memory CD8 T cell and CD14 myeloid populations. Transcriptomic interrogation of these populations further revealed a significant number of differentially expressed genes between the blood and SF with several novel gene pathways unique to the SF. The authors generated a high-resolution map of the immune cell makeup of the SF in oligo-PsA using CyTOF and combined this approach with a cell-specific transcriptomic interrogation of expanded synovial populations to identify novel inflammatory models in PsA.

Anna Antony (Bath, UK) assessed the association between psoriatic nail dystrophy and radiographic damage in 134 patients with PsA. Data included scored radiographs of hands using the PsA Ratingen or PsA-modified Sharp/van der Heijde score and documentation of nail disease using the Psoriatic Nail Severity Score. Chi-square tests were performed to examine the association between the presence of psoriatic nail dystrophy and radiographic damage at the distal interphalangeal joints (DIP) and all other joints (AOJ), as well as to assess the association between nail dystrophy and radiographic damage at the DIP and proximal interphalangeal joints/metacarpophalangeal joint (PIP/MCP) of the corresponding digit. Nail dystrophy was present in $70.1 \%$ of patients, and $90 \%$ of patients had radiographic damage. There was a significant association between the presence of nail dystrophy and erosive disease at the DIP and AOJ. In individual digits, there was an association between onycholysis, hyperkeratosis, and severe nail disease and erosive disease in the corresponding DIP $(\mathrm{p}<0.05)$. A significant association was found between severe nail disease and erosive disease at the corresponding MCP/PIP. Pitting was only associated with joint space narrowing. The author concluded that the presence of onycholysis, hyperkeratosis, and severe nail disease, but not nail pitting, is associated with erosive arthropathy at the DIP in PsA.

Elziane Cruz Ribeiro e Souza (Uberlândia, Brazil) evaluated the correlation between the PsA Impact of Disease (PsAID) score and MDA and VLDA status. The researchers recruited 150 patients in a cross-sectional study and grouped the patients according to MDA status: MDA (meeting 5/7 MDA criteria), VLDA (meeting 7/7 MDA criteria), and non-MDA. To compare the domains of PsAID among the MDA groups, the Kruskal-Wallis tests and the Dunn test were applied. Overall, $16.7 \%$ achieved MDA, $11.3 \%$ achieved VLDA, and $72 \%$ remained non-MDA. Patients in MDA and VLDA had significantly lower median PsAID total scores than non-MDA patients [MDA 1.7 (interquartile range; IQR 1-3.6), VLDA 0.6 (IQR 0.2-0.9), non-MDA 4.6 (IQR $2.9-6.8) ; \mathrm{p}<0.001]$. Accordingly, statistically significant differences were observed between MDA/VLDA groups and non-MDA patients for all PsAID domains $(\mathrm{p}<0.001)$. PsAID score $<4$ was expressed by $84 \%$ of patients in MDA and $100 \%$ of patients in VLDA versus $42.6 \%$ of non-MDA $(\mathrm{p}<0.0001)$. The author concluded that the PsAID score correlated with the MDA status, and patients in MDA/VLDA had significantly lower effect of the disease as measured by PsAID.

Hannah den Braanker (Rotterdam, the Netherlands) hypothesized that lymphatic endothelial cells (LEC) could control migration of pathogenic Th cells by influencing their homing properties. Primary human dermal LEC, and for comparison, primary fibroblast-like synoviocytes (FLS) of a patient with PsA from a noninflamed joint were preincubated for 3 days with or without SF of patients with PsA. Then, these LEC or FLS were co-cultured with allogeneic memory Th cells of 3 healthy donors with $\alpha \mathrm{CD} 3 / \alpha \mathrm{CD} 28$ stimulation. After $72 \mathrm{~h}$, co-cultures were analyzed for chemokine receptor (CCR/CXCR), cytokine, signaling molecule, and transcription factor expression. The author found that healthy dermal LEC induce skin homing receptor CCR10 expression and Th22 cell cytokine IL-22 production in memory Th cells. FLS promotes Th17 cell generation by inducing CCR6 and CCR4 expression and IL-17A production. Healthy dermal LEC

Personal non-commercial use only. The Journal of Rheumatology Copyright $\subset$ $\subset$ 2019. All rights reserved 
pretreated with SF induced even more CCR 10 expression on memory Th cells. LEC upregulated the notch ligand delta-like ligand 4 (DLL4) compared to FLS. Blocking DLL4 using a monoclonal antibody in the dermal LEC/memory Th cell co-culture resulted in more CCR6 and CCR4 expression and IL-17A production, indicating promotion of Th17 cells instead of Th22 cells. The author concluded that healthy dermal LEC can influence homing receptors on memory Th cells and skew memory Th cells more to Th22 cells than Th17 cells, whereas blocking DLL4 in the cultures reversed promotion of Th 22 cells in dermal LEC/memory Th cells and resulted in more Th17 generation.

Ali Duarte-Garcia (Rochester, Minnesota, USA) assessed the domain match (distribution of responses, floor/ceiling effects), feasibility, construct validity, reliability, responsiveness, and discrimination of the 66/68 swollen and tender joint counts (SJC66/TJC68) in PsA. Domain match and feasibility were assessed using an international Web-based survey. Distribution and floor/ceiling effects were assessed using data analyzed from 1 longitudinal observational study (LOS) and 7 PsA randomized controlled trials (RCT). Construct validity reported in correlations, reliability by intraclass correlation, responsiveness using standardized response mean, and discrimination using standardized mean difference were assessed by a systematic literature review as well as a secondary data analysis from the LOS and RCT. Of 18 people completing the Web survey (patients and clinicians), 17 agreed that SJC66/TJC68 measured the domain of interest. The author concluded that SJC66/TJC68 is a feasible, valid, and reliable instrument to measure the musculoskeletal disease activity domain in PsA.

Lucy Durham (London, UK) hypothesized that the CD8+ $\mathrm{T}$ cell receptor $\beta$-chain (TCR $\beta$ ) repertoire in PsA SF differs from blood and between IL-17+ and IL-17-CD8+ T cells. To provide evidence for possible (self-) antigen-driven expansion of IL-17+CD8+ T cells in the SF, the TCR $\beta$ repertoire of total memory, IL-17+, and IL-17-CD8+ and CD4+ T cells from paired SF were compared with blood from patients with PsA. Total memory, IL-17+IFN $\gamma \pm$, and IL-17-IFN $\gamma+$ CD4+ and CD8+ T cells were sorted from SF, and memory CD4+ and CD8+ T cells from blood from 4 patients with PsA. DNA was isolated and TCR $\beta$ chains were sequenced by high-throughput sequencing. TCR $\beta$ sequences from bulk CD4+ and CD8+ T cells from healthy control blood were extracted from publicly available databases. The study did not identify increased overall clonal expansion of synovial $\mathrm{T}$ cells compared to blood in PsA. However, the specific CD8+ TCR $\beta$ sequences highly expanded in SF were different from the sequences expanded in blood, implying that, rather than the passive transfer of $\mathrm{T}$ cell clones from blood into SF, CD8+ T cells expressing specific TCR $\beta$ sequences are specifically activated to proliferate in the SF. The author concluded that conservation of highly expanded TCR $\beta$ sequences suggests that SF CD8+ T cells exhibit a high degree of plasticity and differentiate from a common precursor into different cytokine-producing populations.

Jobie Evans (Cambridge, UK) performed a systematic literature review analyzing the prevalence of undiagnosed axial spondyloarthritis (axSpA) in inflammatory bowel disease (IBD) cases based on cross-sectional imaging of the axial skeleton. This study was conducted in line with MOOSE (Meta-analyses Of Observational Studies in Epidemiology) guidelines for observational studies. There were 12 observational studies identified: 8 assessing the prevalence of computed tomography-sacroiliitis in IBD cases and 4 using magnetic resonance imaging data. The studies included a variety of patient demographics, IBD types, and sample sizes. Inflammatory back pain was noted in $7 / 12$ studies, and 4/12 studies included a control group. Only 3/12 studies included a clinical rheumatology assessment, and 1 study restricted the imaging assessment to only patients with inflammatory back pain. The prevalence of sacroiliitis in these studies ranged from $15 \%$ to $68 \%$. The author concluded that axSpA is likely widely underdiagnosed in patients with IBD, and that cross-sectional imaging intended for the assessment of IBD can be used to screen for axSpA.

Lyn D. Ferguson (Glasgow, UK) evaluated the independent association of central obesity assessed by waist circumference with the odds of psoriasis, PsA, and RA after controlling for body mass index (BMI) and other confounding factors. UK Biobank measured BMI and waist circumference in 5075 individuals with psoriasis, 906 individuals with PsA, 5532 individuals with RA, and 490,977 controls. Central obesity was defined as $\geq 88 \mathrm{~cm}$ in women and $\geq 102 \mathrm{~cm}$ in men. Logistic regression was used to calculate the odds of psoriasis, PsA, and RA compared to controls for those with central obesity (categorical) or by waist circumference (continuous), adjusting for age, sex, ethnicity, smoking status, deprivation, physical activity, and BMI. Increased waist circumference was associated with higher odds of psoriasis, PsA, and RA. After adjusting for $\mathrm{BMI}$ and other covariates, the association remained significant in psoriasis and RA.

Amir Haddad (Haifa, Israel) tested the performance of the CONTEST questionnaire in diagnosing PsA compared to the other existing tools. The study required 208 patients with psoriasis from dermatology clinics to complete the PsA Screening and Evaluation tool, Toronto PsA Screen 2, Psoriasis Epidemiology Screening Tool (PEST), and Early Arthritis for Psoriatic Patients questionnaires prior to rheumatologic evaluation. A composite score was calculated by abstracting scores of items from the original tools that constitute the CONTEST questionnaire. The sensitivity and specificity of the different instruments were calculated using the defined cutoffs of each instrument. Of the 208 patients screened, 108 fulfilled the ClASsification for Psoriatic ARthritis (CASPAR) criteria for diagnosis of PsA. The author concluded that all screening questionnaires are helpful in

Personal non-commercial use only. The Journal of Rheumatology Copyright @ 2019 . All rights reserved. 
excluding PsA but cannot serve as substitutes for clinical diagnosis. The CONTEST questionnaire had no additive diagnostic value to the other screening questionnaires, and the PEST questionnaire had the highest sensitivity and specificity.

Joseph Hutton (Cambridge, UK) examined the chronological order in which pathological radiological changes occur in PsA mutilans (PAM) by conducting a retrospective cohort study of 610 patients with PsA. Patients were screened for PAM on their most recent hand and foot radiographs. Radiographs were quantitatively scored for erosions, joint space narrowing, osteopenia, osteoproliferation, and osteolysis using validated scores. Radiographic progression will be assessed using Poisson regression, generalized additive mixed models, and machine-learning methods. Correlation between disease duration and each feature will be assessed by linear mixed models. The proportion of patients demonstrating osteolysis, joint space narrowing, erosions, osteopenia, and osteoproliferation at different cutoff points will be plotted against disease duration. Radiographic damage as determined by both proportion of maximum possible score and maximum possible joint count will be plotted by joint groups against disease duration. Overall, 36 patients met radiographic criteria for PAM (5.9\%). The author hopes that this ongoing study lends new insights into the chronological order in which radiographic changes occur in PAM, and could have important applications in facilitating the early identification and treatment of patients at risk of developing PAM.

Sonam Kiwalkar (Portland, Oregon, USA) compared clinical characteristics and QOL measures between psoriatic spondyloarthritis (PsSpA) and axSpA patients by conducting a Web-based survey of patients from Spondylitis Association of America. PsSpA shares features of both PsA and axSpA, but previous studies have not been consistent about differences in characteristics between PsSpA and axSpA patients. The study included 644 patients with axSpA and 52 patients with PsSpA. Demographic factors were not significantly different between the 2 groups. The PsSpA group was treated with more methotrexate $(\mathrm{p}=0.004)$ and biologics/biosimilars $(\mathrm{p}=0.03)$. Knee pain $(\mathrm{p}=0.048)$, hand pain $(\mathrm{p}=0.001)$, and foot pain $(\mathrm{p}=0.02)$ were significantly more frequent in patients with PsSpA, and these patients had significantly worse QOL scores on all 4 domains of the Evaluation of Ankylosing Spondylitis QOL scale: physical function $(\mathrm{p}=0.001)$, disease activity $(\mathrm{p}=0.005)$, emotional well-being $(\mathrm{p}=0.002)$, and social participation $(\mathrm{p}=0.0002)$. The author concluded that patients with PsSpA have worse QOL compared to patients with axSpA.

Julia Manasson (New York, New York, USA) investigated the expression of vascular adhesion protein 1 (VAP-1) and its soluble form, semicarbazide-sensitive amine oxidase (SSAO), in patients with psoriasis. VAP-1 is an endothelium-expressed ectoenzyme involved in the process of leukocyte extravasation to sites of inflammation through its SSAO activity. Serum samples were collected from 89 psoriatic patients (47 with PsA) and 24 healthy controls to measure SSAO expression. SF samples were obtained from 5 patients with PsA, 46 with OA, and 16 healthy controls to measure VAP-1 expression. SF concentration of SSAO was significantly higher in patients with PsA compared to healthy controls $(p=0.002)$ and patients with OA $(p=0.002)$. Serum concentration of SSAO was significantly lower in psoriasis patients without PsA compared to healthy controls $(\mathrm{p}=0.0035)$ and those with PsA $(\mathrm{p}=0.08)$. In matched samples, serum concentration of SSAO was higher than SF concentration. The author concluded that VAP-1 may play a role in chronic inflammation occurring in PsA synovial tissues.

Lourdes Perez-Chada (Boston, Massachusetts, USA) aimed to develop and establish the content validity of 2 new sleep patient-reported outcome measures intended to characterize sleep in patients with psoriatic disease: the "PsO Sleepy-Q" and the "PsA Sleepy-Q". The author hypothesized a conceptual framework and identified domains through literature review and patient interviews, rated domains for importance by patients and an international expert panel, selected final domains based on patients' preferences, and generated item pools and a preliminary version of the instruments. Psoriatic patients reported that sleep maintenance, adequacy, quantity, and quality were the most important aspects of disordered sleep. Itch, skin and joint pain, stiffness, taking care of lesions before bedtime, worsening of symptoms at night, feeling uncomfortable in bed as a result of PsA, and sleep apnea were the most outstanding causes of disturbed sleep. Patients reported that disturbed sleep may increase psoriatic signs and symptoms, fatigue, cognitive impairment, and psychological stress. Patients with PsA expressed a higher burden of sleep disturbance compared to those without PsA. Preliminary versions of the 2 questionnaires were created, and the author intends to measure the degree of sleep disturbance, the potential causes of sleep disturbance, and the impairment related to sleep disturbance. The author concluded that these questionnaires will undergo further psychometric testing to define the final forms.

Ari Polachek (Toronto, Ontario, Canada) evaluated the disease activity of patients with late-onset PsA (LoPsA) at presentation and during followup, and evaluated prognosis after several years of followup compared to younger-onset PsA (YoPsA) patients. The study included 566 patients from a large longitudinal PsA cohort who were followed prospectively. Patients were divided into 2 groups: (1) LoPsA ( $\mathrm{n}=$ 177), defined as disease onset after age 50; and (2) YoPsA $(n=389)$, defined as disease onset before age 50. Patients were compared at presentation and after 5 years of followup. At presentation, the LoPsA group was characterized by fewer males (OR 0.4, p = 0.001), less HLA-C*06 (OR 0.3, $\mathrm{p}=0.005)$, longer psoriasis duration $(\mathrm{OR} 1.04, \mathrm{p}=0.0005)$,

Personal non-commercial use only. The Journal of Rheumatology Copyright @ 2019 . All rights reserved. 
higher BMI (OR 1.1, p = 0.0005), and higher modified Steinbrocker score (mSS; OR 1.1, $\mathrm{p}=0.005)$ compared to the YoPsA group. After 5 years, the patients with LoPsA showed significantly higher mean mSS compared to the YoPsA group $(\mathrm{p}=0.02)$. Regression adjusted for sex, BMI, psoriasis duration, HLA, and treatments revealed higher mean $\mathrm{mSS}$ in the LoSpA group compared to the YoPsA group (OR 13.39, $\mathrm{p}=0.007$ ). The author concluded that patients with LoPsA are characterized at presentation by female predominance, higher BMI, more damage, and less HLA-C*06. These patients also have a worse prognosis after 5 years of followup.

Aaida Samad (Cleveland, Ohio, USA) assessed adiposity in children with juvenile PsA (JPsA) in the Childhood Arthritis and Rheumatology Research Alliance registry and compared this to other patients with juvenile idiopathic arthritis (JIA) and to the US general pediatric population. Patients were divided into non-overweight and overweight groups, and the groups were assessed for differences in demographics, family history, clinical characteristics, radiologic manifestations, and scores of health assessment questionnaires at baseline and after 1 year of followup. Overweight children represented $36.3 \%$ of this JPsA cohort. Compared to non-overweight children, they were significantly older at symptom onset and rheumatologist's first assessment, and they scored significantly worse on patient/physician outcome measures. At 1 year, changes in BMI were not associated with changes in clinical features or outcome measures. The prevalence of overweight and obesity in JPsA patients was significantly higher than in other JIA patients and the US general pediatric population. The author concluded that almost 1 in 5 patients with JPsA are obese and that more than one-third are overweight.

Curtis Sobchak (Toronto, Ontario, Canada) assessed whether subclinical atherosclerosis as evaluated by carotid ultrasound (US) could predict future cardiovascular events (CVE) in patients with psoriatic disease, and whether incorporation of imaging data could improve the properties of clinical cardiovascular algorithms. Patients with psoriatic disease $(n=607)$ underwent US assessment of the carotid artery at baseline. The extent of atherosclerosis was assessed using mean carotid intima-media thickness (cIMT) and total plaque area (TPA). Patients were followed at 6- to 12-month intervals, and incident CVE were identified and classified as major CVE or minor CVE. Overall, 37 patients developed incident CVE (28 major CVE). The incidence of CVE was higher in patients with bilateral carotid plaques compared to those with unilateral or no plaques $(\mathrm{p}<0.001)$. In multivariable regression models, TPA (HR 2.20, p < 0.001), mean cIMT (HR 1.21, p < 0.001), and bilateral carotid plaques (HR 2.89, $\mathrm{p}=0.03$ ) predicted incident CVE. The author concluded that psoriatic disease patients with subclinical carotid atherosclerosis are more likely to develop future incident CVE and that carotid US could potentially improve the identification of patients with psoriatic disease who are at high $\mathrm{CV}$ risk.

Dilek Solmaz (Ottawa, Ontario, Canada) studied the prevalence of MDA components in patients with PsA and evaluated disease characteristics and patterns in patients with or without MDA (5/7 MDA criteria). The study used a prospective, multicenter, Web-based registry and included PsA patients who had at least 1 year of disease duration and had full data for MDA $(n=317)$. There were no differences in demographics between groups. The monoarthritis subtype (RR 2.01), absence of enthesitis (RR 1.57), and absence of DIP joint disease (RR 1.1) were associated with higher probability of achieving MDA. For patients achieving MDA, pain visual analog scale and patient's global assessment domains could less frequently be achieved. For patients who did not achieve MDA, body surface area and SJC domains could more frequently be achieved. The author concluded that MDA criteria provide an objective target for treatment in trials and clinical practice, patient-reported outcomes are the most significant barrier to achieving MDA within all domains, and monoarthritis subtype is a good prognostic factor to achieve MDA.

Margot Van Mechelen (Leuven, Belgium) studied the early events leading to PsA by combining a protocol of forced exercise in mice with simultaneous locally induced cutaneous or intestinal inflammation. In the study, 8-week-old C57/B16 male mice were treated with IMQ cream on back skin or with dextran sodium sulphate (DSS) dissolved in drinking water. Control mice were left untreated. Half the mice were then subjected to forced treadmill running to increase biomechanical stress. Assessment of skin and intestine confirmed the presence of local inflammation in the treated mice, and there was evidence of secondary systemic inflammation. Histologic assessment of the joints revealed mild synovitis and enthesitis in the IMQ- or DSS-treated mice. Further analysis showed that forced exercise further boosts this inflammation despite leading to slight improvement in cutaneous and intestinal inflammation. The author concluded that local induction of psoriasis or IBD-like inflammation also triggers a systemic inflammatory response with bone loss and signs of joint diseases, and that forced exercise increases the degree of synovitis and enthesitis. This supports the hypothesis that biomechanical stress contributes to disease manifestations in PsA.

Leonieke J.J. van Mens (Amsterdam, the Netherlands) assessed whether secukinumab modulates the immunopathology of target lesions without blunting systemic immune responses by using peripheral spondyloarthritis $(\mathrm{pSpA})$ as a model. The study included 20 active $\mathrm{pSpA}$ patients in a 12-week open-label trial with secukinumab (300 mg weekly for 4 weeks followed by every 4 weeks). The efficacy endpoint (ACR20) response at Week 12 was achieved by $13 / 20$ patients with rapid and significant improvements in all clinical disease activity measurements.

Personal non-commercial use only. The Journal of Rheumatology Copyright (C) 2019. All rights reserved. 
Clinical improvement in joint counts was associated with histological decrease in synovial sublining macrophages $(\mathrm{p}=0.028)$ and neutrophils $(\mathrm{p}=0.004)$, sensitive synovial biomarkers of inflammatory response in $\mathrm{pSpA}$, as well as with decreased synovial expression of IL-17A $(p=0.010)$ but not TNF. With the exception of IL-17A, the capacity of peripheral blood cells to produce a broad panel of cytokines and chemokines upon stimulation with microbial antigens was not affected. The author concluded that clinical improvement with secukinumab treatment is paralleled by immunomodulation of the inflamed target tissues without compromising systemic immune responses in $\mathrm{pSpA}$.

Kim Wervers (Rotterdam, the Netherlands) evaluated the effect of time to MDA on health-related QOL (HRQOL) at 1 -year followup in patients with newly diagnosed PsA. PsA patients with a new diagnosis of PsA and not yet treated for PsA with disease-modifying antirheumatic drugs were included. MDA status was determined every 3 months in the first year. Medical Outcomes Study Short Form-36 physical component scores (SF-36-PCS) and mental component scores were used to assess HRQOL. Of 296 patients who had had their 1-year visit, 96 (32\%) were in MDA within 3 months (early MDA), 78 (26\%) between 3 and 12 months (late MDA), 98 (33\%) were not in MDA (no MDA), and 24 (8\%) had missing data. Late MDA and no MDA patients had significantly higher baseline TJC, enthesitis scores, and visual analog scores than early MDA patients. At baseline, SF-36-PCS scores were significantly lower in the late and no MDA groups, but after 1 year, only the scores of the no MDA group were significantly lower. The author concluded that patients not in MDA in their first year after diagnosis had significantly worse HRQOL than patients in MDA, but HRQOL of early and late MDA patients did not differ at 1 year.

Yan Zhou (Sacramento, California, USA) assessed transepidermal water loss (TEWL) using quantitative instrumentation, epidermal hyperplasia (by H\&E staining), dermal inflammatory infiltrate (by Giemsa and immunohistochemical staining), and Th17-related genes (by qRT-PCR) in wild type (WT) and TRPVI and TRPAl gene knockout (KO) mice following daily application of topical IMQ cream for 5 days. A role for TRPV1 in PsD has previously been described, but the role of TRPA1 in dermal inflammation and epidermal function is unclear. In this study, TEWL scores were significantly decreased in TRPV1 KO mice by $50.07 \%$ and in TRPAl KO mice by $54.36 \%$, compared with WT mice, suggesting a reduction in IMQ-mediated barrier defects. The fold change of epidermal hyperplasia was decreased in both KO mice compared with WT mice. Additionally, the area of epidermal Munro (neutrophilic) microabscesses was decreased in both KO compared with WT mice, suggesting that neutrophil recruitment was affected in the $\mathrm{KO}$ mice. Lastly, mast cells as well as CD31+ blood vascular cells, CD45+ leukocytes, and CD3+ T cells were all reduced in the lesional skin of KO mice. These results show that dermal inflammation is reduced in TRPV1 $\mathrm{KO}$ mice as expected and that the contributory role of TRPA1 in dermal inflammation is stronger than that of TRPV1. The author concluded that key markers for psoriatic inflammation are reduced in TRPA1 KO mice, which suggests a novel role for TRPA1 in psoriasiform inflammation and a new avenue for therapeutic intervention.

GRAPPA members were enthusiastic in their appreciation of the trainees' work and encouraged them to continue. The next GRAPPA Trainees Symposium will be held in July 2019 in Paris, France.

\section{REFERENCES}

1. Coates LC, Ritchlin CT. GRAPPA Trainees Symposium 2009: a report from the GRAPPA 2009 annual meeting. J Rheumatol 2011;38:526-9.

2. Ritchlin CT. GRAPPA Trainees Symposium 2010: a report from the GRAPPA 2010 annual meeting. J Rheumatol 2012;39:394-7.

3. Ash Z, Ritchlin CT. GRAPPA Trainees Symposium 2011: a report from the GRAPPA 2011 annual meeting. J Rheumatol 2012;39:2184-8

4. Garg N, Touma Z, Ritchlin CT. GRAPPA Trainees Symposium 2012: a report from the GRAPPA 2012 annual meeting. J Rheumatol 2013;40:1413-8.

5. Szentpetery A, Johnson MA, Ritchlin CT. GRAPPA Trainees Symposium 2013: a report from the GRAPPA 2013 Annual Meeting. J Rheumatol 2014;41:1200-5.

6. Eder L, Tillett W, Ritchlin CT. GRAPPA Trainees Symposium 2014: a report from the GRAPPA 2014 Annual Meeting. J Rheumatol 2015;42:1016-20.

7. Milliken M, Generali E, Marin J, Ritchlin CT. GRAPPA Trainees Symposium 2015: a report from the GRAPPA 2015 Annual Meeting. J Rheumatol 2016;43:952-8.

8. Polachek A, Stober CB, Gudbjornsson B, Ritchlin CT. GRAPPA Trainees Symposium 2016: a report from the GRAPPA 2016 Annual Meeting. J Rheumatol 2017;44:661-7.

9. Furer V, Manasson J, Boehncke WH, Ritchlin CT. GRAPPA Trainees Symposium 2017: A Report from the GRAPPA 2017 Annual Meeting. J Rheumatol Suppl 2018;94:4-10. 